\title{
Optical fiber vacuum sensor based on modal interferometer and PDMS coating
}

\author{
Joaquin Ascorbe, Omar Fuentes, Jesus M. Corres Member, IEEE, Francisco J. Arregui, Member, IEEE and Ignacio R. \\ Matias Senior Member, IEEE \\ Department of Electrical, Electronic and Communication Engineering, Public University of Navarra \\ Institute of Smart Cities, Public University of Navarra \\ Pamplona, SPAIN
}

\begin{abstract}
This work studies the behavior of polydimethylsiloxane (PDMS) as a transducer for optical vacuum pressure measurements. The optical structure chosen for this device is a modal interferometer achieved by splicing a coreless multimode optical fiber between two single mode fibers. Then, an etching process is applied to the obtained device, in order to decrease the diameter of the fiber and increase the sensitivity. Finally, the fiber is coated by dip-coating with a layer of PDMS, which changes its volume with pressure changes. The device has been studied in the $1 \times 10^{-3}$ mbar to 10 mbar range with a wavelength shift of $4 \mathrm{~nm}$. A maximum sensitivity of $35 \mathrm{~nm} / \mathrm{mbar}$ was obtained. The simple fabrication process, which can be applied to other structures more sensitive to strain, suggest that PDMS can be a good choice for the development of optical fiber vacuum sensors.
\end{abstract}

Keywords—optical fiber; modal interferometer; vacuum sensor; polydimethylsiloxane; PDMS;

\section{INTRODUCTION}

Vacuum measurement is important in several application fields, such as industry, medical care, energy production, physical experiments and others [1], and many types of sensors have been developed. For vacuum measurement, one of the most extended technology is Pirani gauge, which is based on the heat transfer between a heated wire and the vacuum environment [2]. Pirani gauges allows to measure pressure up to $1 \times 10^{-4} \mathrm{mbar}$. Smaller pressures require other kind of gauges, such as cold cathode or hot cathode gauges, which allows to measure pressures up to $1 \times 10^{-9}$ and $1 \times 10^{-12}$ mbar, respectively [3].

However, this kind of sensors might have a limited usage in some applications, such as heavy industrial environments. Then, the advantages of optical fiber sensors, specially their electromagnetic immunity and their size, can help in these hazardous environments. Among optical fiber structures utilized for vacuum pressure sensing, different configurations and working principles can be found. Fabry-Pèrot interferometers have been fabricated using a vacuum-sealed cavity whose length varies as a function of the pressure [4]. There, the diaphragm is fabricated following a process that includes several steps, such as photoresist patterning and reactive ion etching (RIE). Another optical structure appropriate for vacuum sensing is fiber Bragg gratings (FBGs) [3] [5], which shows high sensitivity to strain and temperature. Here, the working principle is similar to that one of the Pirani gauge, since the authors heat the metal-coated

This work was supported by Spanish Agencia Estatal de Investigación (AEI). Fondo Europeo de Desarrollo Regional (FEDER) (TEC2016-79367-C22-R and TEC2016-78047-R).
FBG by launching a laser light inside the FBG [5] and measure the difference of the transferred heat.

In this paper, we propose a novel method to develop optical fiber vacuum sensors based on the strain applied to a single mode - multimode - single mode (SMS) fiber interferometer. This structure has been chosen for its simplicity of fabrication. The SMS structure generates a modal interferometer, which is sensitive to several parameters, such as temperature [6], strain [7]-[10] or refractive index [11]. Besides, it has been previously demonstrated that reducing the diameter of the fiber, generally by chemical etching, leads to an increase of the sensitivity [12]. In addition, for this particular structure, decreasing the diameter increases the strain produced by the coating and leads to an increase of the sensitivity. The coating is made of polydimethylsiloxane (PDMS), coated by dip-coating and cured at atmospheric pressure. Due to its elastic properties, vacuum pressure makes PDMS to expand and, consequently, it transmits this deformation to the fiber.

The paper is structured as follows: firstly, in section II the fabrication details and the experimental set-up are described. Section III shows the experimental results related to device fabrication and vacuum pressure measurements. Finally, in section IV some conclusions remarks are commented.

\section{FABRICATION PROCESS}

The length and diameter of the MMF segment are the critical parameters for the design of the device. The first step to fabricate this device is to cut a $15 \mathrm{~mm}$ length piece of multimode coreless optical fiber (POFC, Inc.) at $90^{\circ}$ in both sides. Its length was of $15 \mathrm{~mm}$, because it permits to obtain a good interference pattern when decreasing the diameter [11]. Then, this segment was spliced to two standard SMF from Telnet, Inc. The diameter of all fibers is $125 \mu \mathrm{m}$. Then, this structure was etched using hydrofluoric acid (HF) until the diameter of the fiber was reduced to $30 \mu \mathrm{m}$. Then, the etched SMS was washed with water to remove all the HF. This process was monitored to stop it when the transmitted optical spectrum reaches the desired characteristics: a quasi- sinusoidal spectrum. This behavior facilitates the shift-phase study.

Finally, the resultant device was glued to a U-holder and then it is coated with PDMS Sylgard 184 [13]. PDMS was prepared by mixing the elastomer base with the curing agent in 10:1 proportion. After that, the mix was solved in toluene (1:1), to 
obtain a lower viscosity solution, which will later provide greater control on the thickness and shape of the coating, made by dip-coating. Just after coating, the device is heated to $100^{\circ} \mathrm{C}$ to dry the layer at atmospheric pressure. The final thickness obtained was $1.5 \mu \mathrm{m}$.

The experimental set-up used for the characterization of this device, shown in Fig. 1, is the typical transmission set-up, consisting of a light source (Agilent 83437A) and an optical spectrum analyzer (HP 86142A). The device was placed inside a vacuum chamber, which has an optical feedthrough, where the vacuum is achieved thanks to a rotary vacuum pump and a turbomolecular pump. The vacuum pressure was acquired using a data-logger (Agilent 34972A) and a vacuum pressure sensor (Pfeiffer PKR251), which consists of a Pirani gauge and a coldcathode gauge.

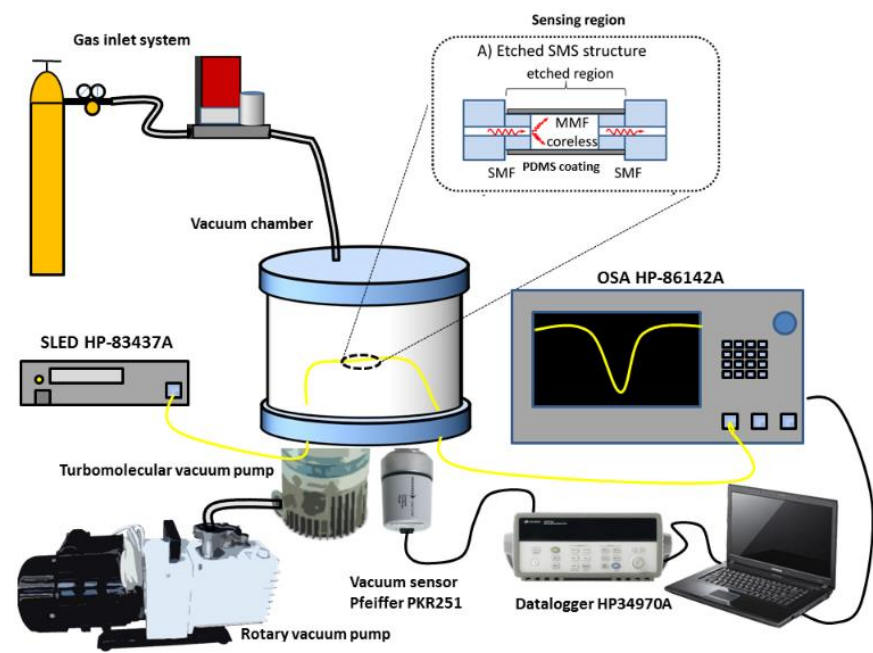

Fig.1. Experimental set-up.

\section{EXPERIMENTAL RESULTS}

As it is well known, the SMS interferometers can be tuned to work in two modes of operation. One of this is the self-image effect [14], [15], which produces a maximum in the transmission spectrum that is distinguished from other maxima obtained by a fractional constructive interference. To obtain the location of said maximum wavelength [16], the following expression (1) was used:

$$
\lambda=\frac{4 D^{2} n}{Z}
$$

where $\lambda$ is the operational wavelength, $D$ is the diameter of the MMF section, $n$ is the refractive index of silica and $\mathrm{Z}$ is the MMF segment length. The second mode of operation of the SMS structure permits to use it as both a wavelength and a phase sensitive device.

The spectrum evolves as follows: the attenuation bands blueshift and more bands start to appear from greater wavelengths, which will also suffer a blueshift. Then, those attenuation bands trend to separate and, finally, they start to lose amplitude, but they get a quasi-sinusoidal shape. Adding the PDMS layer redshifts the spectrum.

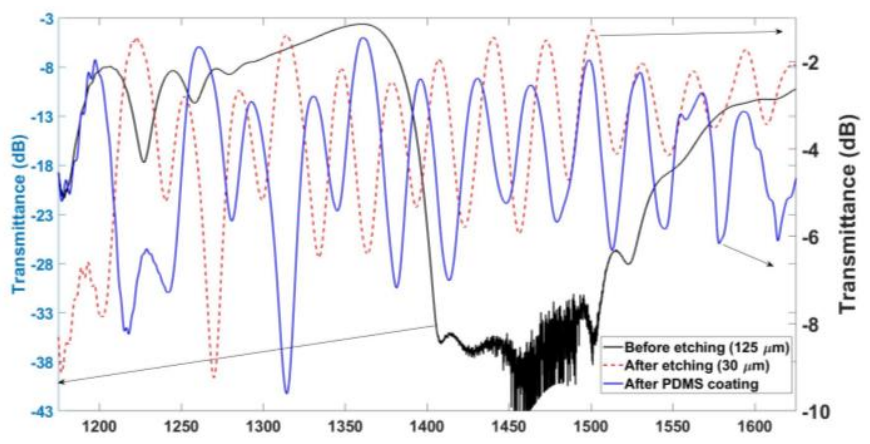

Fig.2. Transmitted optical spectrum at the different steps of the fabrication process.

\section{A. Pressure measurement}

For pressure measurements, the procedure followed is the next: the rotary vacuum pump was started first and the pressure drops up to $3 \times 10^{-1}$ mbar; then the turbo-molecular was turned on until vacuum pressure reaches values near $1 \times 10^{-3}$ mbar. For going back to the atmospheric pressure, vacuum pumps were turned off in the reverse order and then a gas flow was used to final recovering.

For faster optical measurements, the span of the optical spectrum has been reduced and the measurements were performed around $1310 \mathrm{~nm}$. Fig. 3 shows the dynamic response of the optical fiber vacuum sensor as the pressure changes. The left axis corresponds to the wavelength of a peak, whereas the right axis represents the decimal logarithm of the pressure in mbar.

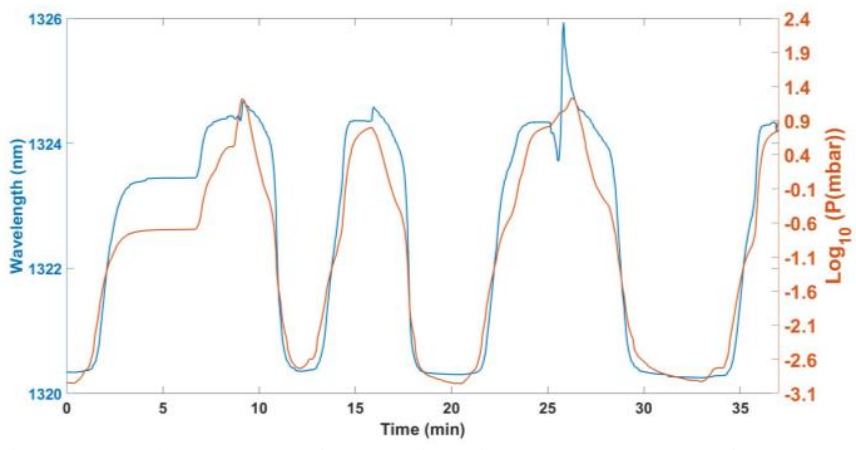

Fig.3. Dynamic response of the optical fiber vacuum sensor for pressure ranging from 16 mbar to $1.1 \times 10^{-3} \mathrm{mbar}$

Analyzing previous figure, Fig. 3, it can be observed that the sensor has good reversibility as well as a high stability. It is also possible to see that there is a peak when the rotary pump is started, which is also present on the commercial sensor. Finally, it is quite clear that the range of this sensor is lower than that one of the commercial sensor. All these aspects can be seen more clearly in the following figure, Fig. 4, where it is represented the wavelength shift as a function of the vacuum pressure. 


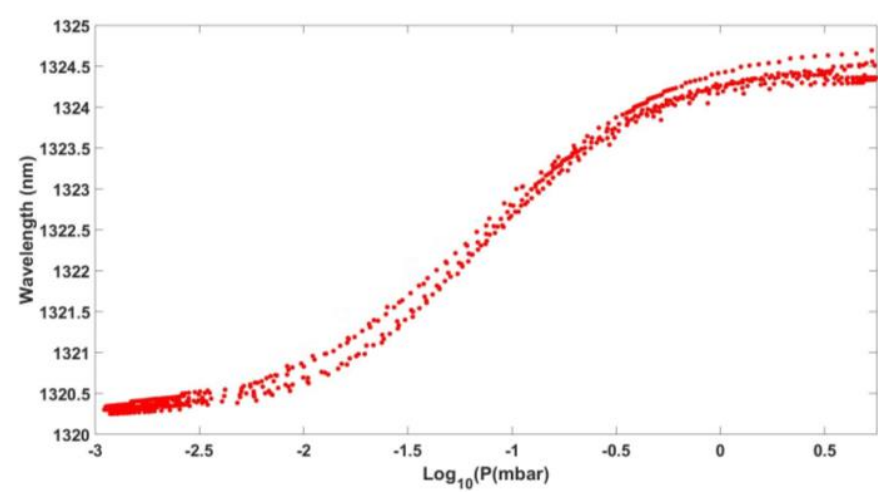

Fig. 4. Wavelength shift of an attenuation band as a function of the vacuum pressure.

In Fig. 4, it is possible to observe how the resonance wavelength blueshifted as the pressure decreases. The greater sensitivity is obtained in the 0.01 to 0.3 mbar range, where the device shows a linear behavior. For greater or smaller pressures, the PDMS layer does not cause the same stress to the fiber and, therefore, the sensitivity decreases.

\section{CONCLUSION}

A novel optical fiber vacuum sensor has been developed using a strain sensitive modal interferometer known as SMS. This sensor is different from other optical sensors dedicated to pressure, which are generally based on membranes and FabryPèrot interferometers or they are based on thermal effects.

This sensor has demonstrated a good behavior on the $1 \times 10^{-3}$ to $1 \mathrm{mbar}$ range. It shows a dynamic range of $4 \mathrm{~nm}$, although the greater change occurs at the 0.01 to $0.3 \mathrm{mbar}$ range. The maximum sensitivity is $35 \mathrm{~nm} / \mathrm{mbar}$. This structure has been chosen for its simplicity of fabrication and for its sensitivity to strain. However, there are optical structure with greater sensitivities, which might offer the possibility to work in other pressure ranges.

It is quite clear that there are other alternatives to modify the sensitivity and range of this kind of sensors. For example, the most immediate option is to study the different base/curing agent ratios, which will affect to the cross-linking degree and, consequently, to the PDMS stiffness.

\section{ACKNOWLEDGMENT}

Joaquin Ascorbe and Omar Fuentes would like to thank the Public University of Navarra, for the post-doctoral and predoctoral Research grant.

\section{REFERENCES}

[1] P. Roriz, O. Frazão, A. B. Lobo-Ribeiro, J. L. Santos, and J. A. Simões, "Review of fiber-optic pressure sensors for biomedical and biomechanical applications," J. Biomed. Opt., 2013.

[2] W. J. Alvesteffer, D. C. Jacobs, and D. H. Baker, "Miniaturized thin film thermal vacuum sensor," J. Vac. Sci. Technol. A, vol. 13, no. 6, pp. 2980 $2985,1995$.

[3] F. Urban, J. Kadlec, R. Vlach, and R. Kuchta, "Design of a pressure sensor based on optical fiber Bragg grating lateral deformation," Sensors, vol. 10, no. 12, pp. 11212-11225, 2010.

[4] K. . H. Y.; Totsu and M. Esashib, "Vacuum Sealed Ultra Miniature FiberOptic Pressure Sensor Using White Light Interferometry," in 12th International Conference on Solid-State Sensors, Actuators and Microsystems, 2003, pp. 931-934.

[5] B. McMillen, C. Jewart, M. Buric, K. P. Chen, Y. Lin, and W. Xu, "Fiber Bragg grating vacuum sensors," Appl. Phys. Lett., vol. 87, no. 23, pp. 13, 2005.

[6] S. Silva et al., "Ultrahigh-sensitivity temperature fiber sensor based on multimode interference.," Appl. Opt., 2012.

[7] R. M. André, C. R. Biazoli, S. O. Silva, M. B. Marques, C. M. B. Cordeiro, and O. Frazão, "Multimode interference in tapered single modemultimode-single mode fiber structures for strain sensing applications," in OFS2012 22nd International Conference on Optical Fiber Sensors, 2012.

[8] A. M. Hatta, Y. Semenova, Q. Wu, and G. Farrell, "Strain sensor based on a pair of single-mode-multimode-single-mode fiber structures in a ratiometric power measurement scheme," Appl. Opt., 2010.

[9] Q. Wu, Y. Semenova, P. Wang, A. M. Hatta, and G. Farrell, "Experimental demonstration of a simple displacement sensor based on a bent single-mode-multimode-single-mode fiber structure," Meas. Sci. Technol., 2011.

[10] A. Mehta, W. Mohammed, and E. G. Johnson, "Multimode interferencebased fiber-optic displacement sensor," IEEE Photonics Technol. Lett., 2003

[11] Y. Cardona-Maya, I. Del Villar, A. B. Socorro, J. M. Corres, I. R. Matias, and J. F. Botero-Cadavid, "Wavelength and Phase Detection Based SMS Fiber Sensors Optimized with Etching and Nanodeposition," J. Light. Technol., 2017.

[12] A. B. Socorro et al., "Fiber-Optic Immunosensor Based on an Etched SMS Structure," IEEE J. Sel. Top. Quantum Electron., 2017.

[13] SYLGARDTM 184, "Technical Data Sheet, The Dow Corning Company."

[14] O. Fuentes et al., "Increasing the Sensitivity of an Optic Level Sensor with a Wavelength and Phase Sensitive Single-Mode Multimode Single-Mode Fiber Structure,” IEEE Sens. J., 2017.

[15] S. Silva et al., "Ultrahigh-sensitivity temperature fiber sensor based on multimode interference," Appl. Opt., vol. 51, no. 16, pp. 3236-3242, 2012.

[16] L. B. Soldano and E. C. M. Pennings, "Optical Multi-Mode Interference Devices Based on Self-Imaging: Principles and Applications," J. Light. Technol., 1995. 